\title{
Refractive index profile optimisation for the design of optical fibres
}

\author{
R. W. Smink • B. P. de Hon • M. Bingle • R. Mussina • \\ A. G. Tijhuis
}

Received: 1 September 2008 / Accepted: 7 April 2009 / Published online: 20 May 2009

(C) The Author(s) 2009. This article is published with open access at Springerlink.com

\begin{abstract}
Owing to advanced manufacturing techniques, it is possible to produce cylindrical single-mode fibres with nearly arbitrary refractive index profiles. For the design of optical fibres automated optimisation schemes have yet to be exploited. We have employed deterministic local, and stochastic global optimisation schemes for the minimisation of a cost function based on dispersion, dispersion slope, macro-bending losses and mode-field diameter, on the space of continuous piecewise linear dopant concentration profiles. For the local schemes (modified and quasi Newton), it appears possible to select a few initial profiles, such that the optimisation results are close to the "global optima" (within 8\%), found using global schemes (simulated annealing and differential evolution), while reducing computation times significantly (minutes instead of days). For the local schemes, the cost function gradient is required. Fréchet derivatives are more efficient than finite-difference approximations. A sensitivity analysis provides useful information for manufacturers regarding the required profile accuracy. A comparison of our optimised fibre designs with commercially available optical fibres demonstrates that existing fibres can be improved.
\end{abstract}

Keywords Single-mode optical fibres $\cdot$ Optimisation $\cdot$ Sensitivity analysis $\cdot$ Numerical modelling

\section{Introduction}

When it comes to data transmission rates, optical-fibre based communication links have long topped the leader board, and will do so for the foreseeable future. After the introduction of the multi-mode fibre the first hurdle that had to be taken was to address the excessive propagation

R. W. Smink · B. P. de Hon $(\varangle) \cdot$ R. Mussina $\cdot$ A. G. Tijhuis

Faculty of Electrical Engineering, Eindhoven University of Technology, P.O. Box 513,

5600 MB Eindhoven, The Netherlands

e-mail: b.p.d.hon@tue.nl

M. Bingle

EM Software \& Systems-SA(Pty) Ltd., P.O. Box 1354, Stellenbosch, 7599, South Africa 
losses, due to impurities in the glass. Once high-quality glass became available, multi-mode dispersion became the bottleneck. With the advent of single-mode fibres, the spectre of dispersion could be greatly reduced. The first single-mode fibre, which is still called the standard single-mode fibre was a so-called step-index fibre, referring to the dependence of the (square of the) refractive index on the radial direction.

Owing to improved manufacturing techniques, like the PCVD process, in which thin glass layers are deposited with the aid of a moving microwave resonator, it is now possible to produce nearly all refractive index profiles. This flexibility in profile manufacturing has provided the opportunity of designing optical fibres with desired properties. Often, such properties are conflicting, e.g. minimum dispersion and dispersion slope are at odds with minimum bending losses. In addition, one would like to keep the mode-field diameter fixed. From a design point of view, manual tuning of the refractive index profile of a single-mode optical fibre to meet specific demands is a painstaking and arduous task.

Fortunately, automated optimisation schemes can speed up this design step considerably. In other fields of research such schemes often lead to counter-intuitive designs that could not have been contrived otherwise. With the odd exception, automated optimisation schemes have not yet been used for the design of axi-symmetric single-mode optical fibres. In this paper, we build on initial fibre optimisation ideas explored by Bingle et al. (2001).

Usually, an optimisation problem is formulated in terms of the minimisation of a cost function. The specific form of this cost function is important, since it not only determines the relative importance of the individual fibre quantities, but it also influences the rate of convergence to a minimum. Likewise, the selection and number of optimisation parameters, viz. the parameters that define the refractive index profile, influence the efficiency and the result of the search substantially. Although many algorithms are available to perform the optimisation, by and large, they can be subdivided into two categories, viz. global stochastic techniques and deterministic methods based on gradient information.

Deterministic gradient-based optimisers, e.g. Newton algorithms (Gill and Murray 1974; Fletcher and Leyffer 1998), are usually much more efficient than stochastic ones. However, a careful initial (refractive index profile) guess is of vital importance as the minimisation process may otherwise end up in a local minimum instead of the desired global one. Further, one has to compute the gradients of the objective function to all optimisation parameters at each iteration step in an efficient way. Of course, these gradients can be estimated by means of a finite-difference approximation, although the optimisation is faster if one uses the actual gradients in terms of Fréchet derivatives.

Statistically oriented optimisation techniques, on the other hand, are generally geared towards finding the global minimum and only require cost function evaluations. A major drawback forms the computation time, which can be extremely long, and therefore severely hampers a flexible design process. Also the initialisation of these routines is delicate, as various control parameters have to be set carefully. Simulated annealing (SA), particle swarm techniques, and several varieties of genetic algorithms, like differential evolution (DE) methods, are typical examples that belong to this class of optimisers (Kirkpatrick et al. 1983; Goffe et al. 1994; Storn and Price 1997; Weile and Michielssen 1997; Rahmat-Samii and Michielssen 1999).

We have performed profile optimisations with two deterministic gradient-based optimisers, viz. a modified-Newton (MN) algorithm E04KDF (Numerical Algorithms Group Ltd 2001) and a quasi-Newton (QN) algorithm (Fletcher and Leyffer 1998; Fletcher 2006), and two global ones, viz. the DE (Mishra 2006a) and SA (Goffe et al. 1994) schemes. The choice of the latter two schemes is based on two articles by Mishra (2006a,b). 


\section{Cost function, optimisation parameters and initial profile}

The cost function (CF) is the function that will be minimised to obtain an optimal refractive index profile. The quality of an optical fibre is characterised in terms of fibre quantities denoted as $v_{i}, i=1, \ldots, M$. We express the $\mathrm{CF}$ as a sum of squared errors, i.e.

$$
\mathrm{CF}=\sum_{i=1}^{M} \alpha_{i}\left[v_{i}\left(x_{n}\right)-v_{i}^{\mathrm{ref}}\right]^{2} .
$$

The desired value for a fibre quantity is denoted by the superscript 'ref'. Scale factors $\alpha_{i}$ have been added to scale the pertaining quantities to the same order, or to stress the importance of a certain quantity above others.

Optimisation with a stochastic technique carries out repeated evaluations of the CF of Eq. 1. For the gradient-based algorithms, gradient information accelerates the search for the minimum of the $\mathrm{CF}$ by providing the direction of steepest descend. Hence, the first derivatives of the $\mathrm{CF}$ with respect to the optimisation parameters $x_{n}$ are required, i.e.

$$
\partial_{x_{n}} \mathrm{CF}=2 \sum_{i=1}^{M} \alpha_{i}\left[v_{i}\left(x_{n}\right)-v_{i}^{\mathrm{ref}}\right] \partial_{x_{n}} v_{i} .
$$

The accuracy with which these gradients are computed, strongly influences the rate of convergence to a minimum.

\subsection{Discretisation}

So far the definition of the cost function was fairly abstract. An essential step in any optimisation scheme is to define proper optimisation parameters. We consider axi-symmetric optical fibres, and hence we employ a right-handed polar cylindrical system of coordinates $\{\rho, \varphi, z\}$, with a radial coordinate $\rho=r / a$ that is normalised to the core radius, $a$. As it is our desire to optimise the refractive index profile of an optical fibre so as to attain a set design goal, we want to retain an optimum flexibility as to its shape. For the discretisation of the profile in the core region, we have chosen a continuous concatenation of piecewise linear segments, with variable endpoints that serve as the free optimisation parameters. This restriction is sensible, since in practice, such profiles are relatively easy to manufacture.

At the sample points inside the core we allow for a horizontal and a vertical variation, except for the on-axis one $\left(\rho_{0}=0\right)$. Each possible variation is denoted by an optimisation parameter $x_{n}$, with $n \in \mathbb{N}$. Since upward and downward refractive index variations are achieved by adding Germanium and Fluor to the Silica, respectively, each dopant can, in principle, be seen as an optimisation parameter, albeit within certain bounds. To keep the total number of parameters limited, we consider the Fluor concentration to be constant throughout the fibre core. Naturally, this constant value sets the maximum possible depth of a trench in the refractive index profile. In Fig. 1, we have plotted an arbitrary Germanium concentration profile with four sample points, denoted by solid dots.

Another possibility is to regard the permittivity as the vertical optimisation parameter, instead of its dopant building blocks. They are (mildly non-linearly) related through the Sellmeier equation (Hermann and Wiechert 1989). That way, we are not restricted to the currently used dopants, thus generalising the overall optimisation. On the other hand, since the refractive index profile is defined at a wavelength of $\lambda=632.8 \mathrm{~nm}$, and designed with linear segments at that wavelength, the conversion to the desired optimisation wavelength requires 

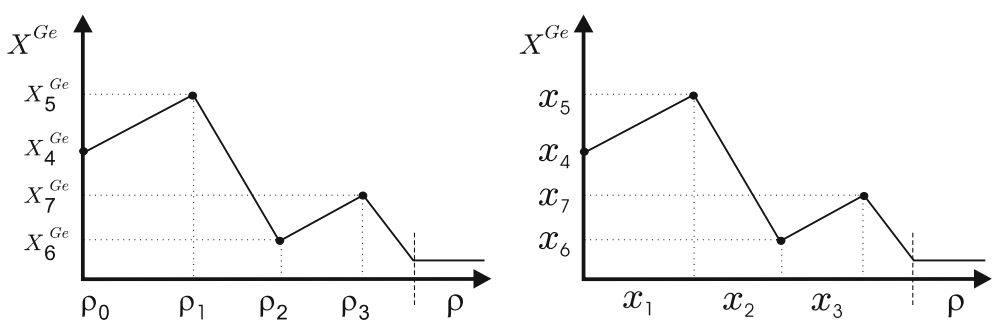

Fig. 1 Free optimisation parameters $x_{n}$ (right) and their relation to the Germanium concentration profile defining variables (left)

additional computation time, and hence optimisation of the Germanium concentration profile is preferred.

In view of our numerical integration scheme, it is necessary to limit the radial range in which to optimise the profile. This limit is set by the core/cladding transition. At the fibre axis, we only permit a vertical variation, implying that the total number of optimisation parameters, $N$, is odd. The optimisation parameters are chosen as follows

$$
\begin{array}{ll}
x_{n}=\rho_{n}-\rho_{n-1}, & \text { for } n=\{1, \ldots,(N-1) / 2\}, \\
x_{n}=X_{n}^{G e}, & \text { for } n=\{(N+1) / 2, \ldots, N\} .
\end{array}
$$

The parameters $x_{n}$ are shown explicitly on the right in Fig. 1. To exclude physically impossible profiles and limit the parameter space, we supplement all the optimisation parameters with simple bounds

$$
\begin{array}{ll}
\varepsilon \leq x_{n} \leq 1-\varepsilon, & \text { for } n=\{1, \ldots,(N-1) / 2\} \\
0 \leq x_{n} \leq 0.5, & \text { for } n=\{(N+1) / 2, \ldots, N\},
\end{array}
$$

and the horizontal parameters with the constraint

$$
\sum_{n=1}^{(N-1) / 2} x_{n} \leq 1-\varepsilon .
$$

The small number $\varepsilon$ is introduced to prevent very steep slopes in the profile, which could lead to numerical difficulties in the gradient computations. The upper bound $X_{n}^{G e}=0.5$, corresponds to a refractive index difference of about $\Delta \approx 3.5 \%$, which we deem large enough for practical applications. Unfortunately, the $\mathrm{MN}$ algorithm available to us can not be supplemented with constraints. For this algorithm, the bounds are chosen in such a way that Eq. 5 can not be violated, thus reducing the parameter space according to

$$
\begin{aligned}
& \varepsilon \leq x_{n} \leq 2(1-\varepsilon) /(N-1), \quad \text { for } n=\{1, \ldots,(N-1) / 2\}, \\
& 0 \leq x_{n} \leq 0.5, \quad \text { for } n=\{(N+1) / 2, \ldots, N\} .
\end{aligned}
$$

The fibre quantities $v_{i}$ are not simple functions of the optimisation parameters, and therefore, we have to evaluate $\partial_{x_{n}} v_{i}$ numerically. From Eq. 3 and Fig. 1, we infer that

$$
\begin{aligned}
& \partial_{x_{n}} F=\sum_{i=n}^{(N-1) / 2} \partial_{\rho_{i}} F, \quad \text { for } n=\{1, \ldots,(N-1) / 2\}, \\
& \partial_{x_{n}} F=\partial_{X_{n}^{G e}} F, \quad \text { for } n=\{(N+1) / 2, \ldots, N\},
\end{aligned}
$$



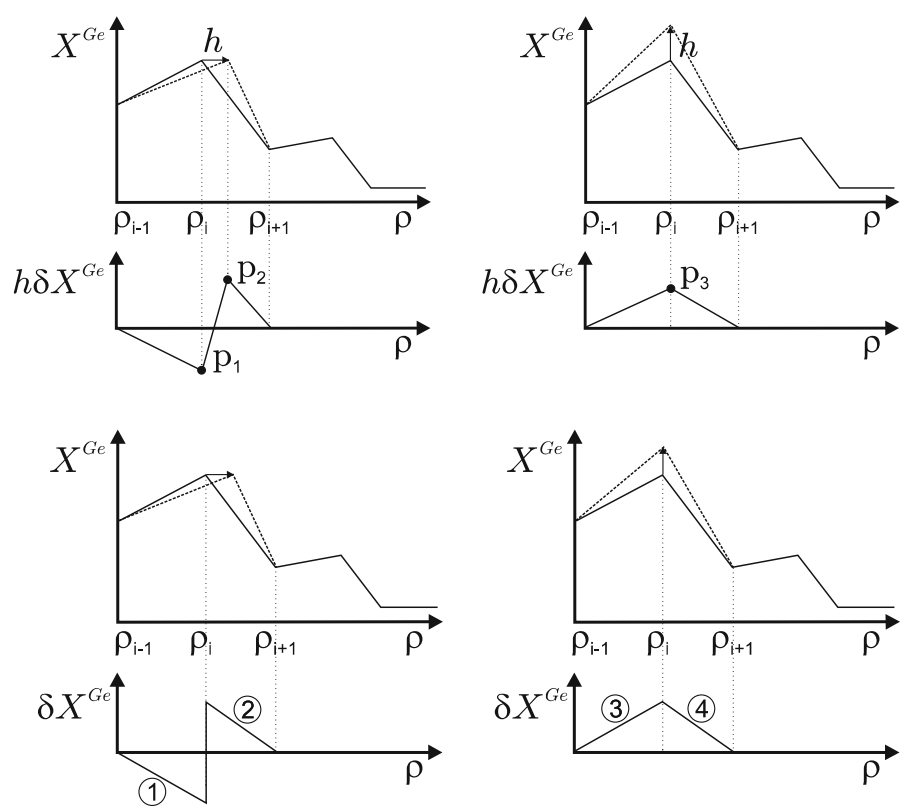

Fig. 2 Horizontal and vertical profile variations and the corresponding profile variation functions $h \delta X^{G e}$ for finite differences (top) and the Fréchet derivative (bottom)

where, for the sake of simplicity, we have introduced the functional $F: D \rightarrow \mathbb{R}$, which corresponds to a single fibre quantity $v_{i}$ (Meyberg and Vachenauer 1991). To obtain the directional derivatives on the right-hand side of Eq. 7, we have applied two approaches, viz. the finitedifference approximation and the Fréchet derivative. The corresponding profile variations are shown at the top and bottom of Fig. 2, respectively. The finite-difference approximation is, wherever physically possible, carried out using a central-difference scheme, and set against the exact computation of the Fréchet derivatives for the various derivatives $\partial_{x_{n}} F$. From this comparison we have concluded that the optimal absolute step sizes for horizontal and vertical variations in the finite-difference scheme are $h=5 \times 10^{-4}$ and $h=5 \times 10^{-5}$, respectively. Thus, an accuracy of six digits is guaranteed. Differentiation in terms of the Fréchet derivatives is more efficient as its computation is a factor two faster than the finite-difference scheme and its accuracy is 12 digits or more, resulting in a faster convergence. On the other hand, the Fréchet derivatives have to be computed by hand, which is a straightforward but laborious affair.

\subsection{The initial profile}

For the gradient-based optimisation, we have to choose an initial profile. Statistically oriented schemes, which do not depend on this choice are used to search for the global minimum of the CF. The results of the global schemes will be set against the (local) minima obtained by the gradient-based optimisers, for a selection of initial profiles. For five optimisation parameters, this selection is shown in Fig. 3. At each equidistant radial point $\rho$ in $\left\{0, \frac{1}{3}, \frac{2}{3}\right\}$ we can choose between two Germanium concentration values, namely $X^{G e}=0 \%$ or $X^{G e}=7 \%$. Hence, each profile consist of three solid dots, which indicate the values of the optimisation 
Fig. 3 Seven possible initial $X^{G e}$ profiles with $X^{F}=0.27 \%$. The dots denote the locations of the optimisation parameters in the $X^{G e}$ profile

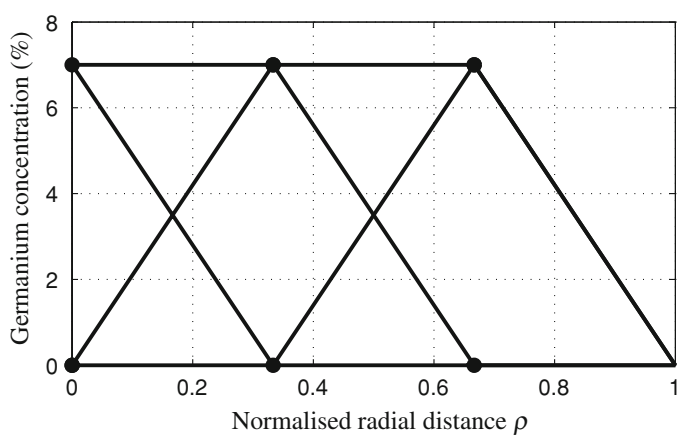

variables. For simplicity, we introduce a binary notation where 0 corresponds to $X^{G e}=0 \%$ and 1 to $X^{G e}=7 \%$. The maximum value of $X^{G e}=7 \%$ is the highest integer value for which the fibre with profile $(1,1,1)$ is still in its (theoretical) single-mode regime. This implies that for an increasing number of optimisation parameters the maximum value of $X^{G e}$ decreases and that the profile approaches that of a step-index fibre. Having discussed the discretisation, we shall briefly discuss how we calculate the elementary optimisation quantities.

\section{The field equations}

Let us consider time-harmonic guided electromagnetic waves with an $\exp (j \omega t)$ time factor. Following de Hon and Bingle (2003), we expand the electromagnetic field components that are tangential to the radial direction in terms of non-dimensionalised field quantities, i.e.

$$
\left(\rho E_{\varphi}, E_{z}, \rho H_{\varphi}, H_{z}\right)=\rho^{|m|} e^{-j m \varphi-j \omega \zeta z / c_{0}}\left(j Z_{0} p_{2},-j Z_{0} p_{1}, q_{1}, q_{2}\right),
$$

in which $m \in \mathbb{Z}$ is the azimuthal index, $\zeta$ is the normalised propagation coefficient, and $Z_{0}$ and $c_{0}$ denote the wave impedance and the speed of light in vacuum, respectively. The radial transmission line equations associated with source-free modal solutions may be cast in the following form (cf. Dil and Blok 1973)

$$
d_{\rho} \mathbf{f}=\rho^{-1} \mathbf{A} \mathbf{f},
$$

in which the field vector is defined through $\mathbf{f}=\mathbf{f}(\rho, m, \zeta, \omega)=\left(p_{1}, p_{2}, q_{1}, q_{1}\right)^{T}$, with the superscript ${ }^{T}$ indicating transposition. For more details, we refer to de Hon and Bingle (2003).

For $m$ and $\omega$ fixed, we may regard Eq. 9 as a singular Sturm-Liouville problem, in that the boundary conditions, imposed on physical grounds are that $\mathbf{f}$ should remain bounded for $\rho \downarrow 0$, while the Sommerfeld radiation conditions for $\rho \rightarrow \infty$ should also be satisfied. The isolated points in the complex $\zeta$-plane for which modal solutions to Eq. 9 exist are the normalised propagation coefficients associated with those modes. These points are zeroes of the characteristic function, $\mathrm{C}(m, \zeta, \omega)$, which is the determinant of four independent field vectors, two of which remain bounded for $\rho \downarrow 0$, while the other two decay exponentially for $\rho \rightarrow \infty$.

Of course, $\mathbf{A}, \mathbf{f}$, and $\zeta$, and hence $\mathrm{C}(m, \zeta, \omega)$ also depend on the refractive index profile. For small $X^{G e}$ variations of the form $X^{G e} \rightarrow X^{G e}+h \delta X^{G e}$, the characteristic function $\mathrm{C} \rightarrow \mathrm{C}+h \delta \mathrm{C}$ must still vanish identically. As a consequence we may write

$$
\delta \mathrm{C}=\left(\partial_{\zeta} \mathrm{C}\right) \delta \zeta+\bar{\delta} \mathrm{C}=0 \Rightarrow \delta \zeta=-\left(\partial_{\zeta} \mathrm{C}\right)^{-1} \bar{\delta} \mathrm{C}
$$


in which $\bar{\delta}$ denotes the first variation while keeping $\zeta$ constant. Upon evaluating the first variation of Eq. 9 with respect to $X^{G e}$, we arrive at

$$
d_{\rho}\left(\begin{array}{c}
\mathbf{f} \\
\bar{\delta} \mathbf{f} \\
\partial_{\zeta} \mathbf{f}
\end{array}\right)=\rho^{-1}\left(\begin{array}{ccc}
\mathbf{A} & 0 & 0 \\
\bar{\delta} \mathbf{A} & \mathrm{A} & 0 \\
\partial_{\zeta} \mathbf{A} & 0 & \mathbf{A}
\end{array}\right)\left(\begin{array}{c}
\mathbf{f} \\
\bar{\delta} \mathbf{f} \\
\partial_{\zeta} \mathbf{f}
\end{array}\right)
$$

which, supplemented with the right boundary conditions is integrated to yield $\partial_{\zeta} \mathrm{C}$ and $\bar{\delta} \mathrm{C}$, and hence $\delta \zeta$. For the computation of the derivatives of $\zeta$ and $\mathbf{f}$ with respect to $\omega$, we have adopted the procedure described in Bingle et al. (2001), which is akin to the one above.

Once the derivatives with respect to $\omega$ and the first variations with respect to $X^{\mathrm{Ge}}$ have been determined, the corresponding derivatives and variations of the optimisation quantities follow upon applying the chain rule. Among the optimisation quantities used in this paper are the dispersion and dispersion slope, respectively, given by

$$
D=-\frac{\hat{\omega}^{2}}{2 \pi a c_{0}}\left(2 \frac{d \zeta}{d \hat{\omega}}+\hat{\omega} \frac{d^{2} \zeta}{d \hat{\omega}^{2}}\right), \quad S=\frac{\hat{\omega}^{3}}{(2 \pi a)^{2} c_{0}}\left(4 \frac{d \zeta}{d \hat{\omega}}+5 \hat{\omega} \frac{d^{2} \zeta}{d \hat{\omega}^{2}}+\hat{\omega}^{2} \frac{d^{3} \zeta}{d \hat{\omega}^{3}}\right)
$$

where $\hat{\omega}=\omega a / c_{0}$ denotes a normalised frequency. Another important optimisation quantity is the macro-bending loss, which is defined in Smink et al. (2007). The final optimisation quantity is the mode-field diameter. The most common definitions are the respective Petermann I and II mode-field diameters, $d_{I}$ and $d_{I I}$, given by

$$
d_{I}^{2}=8 a^{2} \frac{\int_{A_{\infty}} S(\rho) \rho^{3} d \rho d \psi}{\int_{A_{\infty}} S(\rho) \rho d \rho d \psi}, \quad d_{I I}^{2}=32 a^{2} \frac{\int_{A_{\infty}} S(\rho) \rho d \rho d \psi}{\int_{A_{\infty}} \frac{\left[d_{\rho} S(\rho)\right]^{2}}{S(\rho)} \rho d \rho d \psi},
$$

where $S$ denotes the $z$-component of the Poynting vector. In modern optical-fibre data sheets, MFD $d_{I I}$ has superseded MFD $d_{I}$ as the definition of choice. Formally, MFD $d_{I I}$ is only defined for continuous profiles, since $S$ would have a jump discontinuity wherever profile discontinuities occur. Although our profiles are continuous, the first variation in the horizontal direction of the profile is discontinuous (see Fig. 2), implying that extra care has to be taken in the evaluation of the gradient of the mode-field diameter. An alternative definition of MFD $d_{I I}$ in which any discontinuities are simply excluded from the integration produces results that are consistent with finite-difference calculations for which sampling does take place on the manifold of continuous profiles.

\section{Optimised profile results}

Below, we discuss the results of several optimisation runs, showing both the choice of the initial profile, and the number of iterations and the computation time. Finally, we shall perform a sensitivity analysis on the obtained optimised refractive index profiles.

Practical values for the fibre quantities $v_{i}$ depend on the utilisation of the optical fibre. Initially, we have chosen all scale factors $\alpha_{i}$ in the CF of Eq. 1 to be equal to one. Further, we have added a test, which checks whether the fibre is still in its single-mode regime. Although the effective cut-off wavelength would be the appropriate quantity for this check, its computation is too time-consuming inside the optimisation loop. Therefore, we have employed a mode-counting scheme that is based on the theoretical cut-off wavelength (de Hon and Bingle 2003; de Hon 2003) which is much faster, and safely, overestimates the cut-off wavelengths 
of the higher-order modes. If there exists more than one mode, a penalty is added to the CF. Finally, we note that all computations have been performed on a Pentium IV, $2.6 \mathrm{GHz}$ computer.

\subsection{Profile variations in both horizontal and vertical directions}

First, we consider profile variations in both the horizontal and vertical direction at each sample point, with the exception of the on-axis sample point where only a variation in the vertical direction is allowed. We start with optimisation runs consisting of five and seven optimisation parameters. We will give an overview of the end results for profiles with 9, 11, 13 and 15 optimisation parameters.

\subsubsection{Five optimisation parameters}

We have performed optimisation runs at $\lambda=1,550 \mathrm{~nm}$ with the SA and DE schemes and both Newton algorithms for five optimisation parameters. The initial profiles have a core radius of $5 \mu \mathrm{m}$, and are chosen as discussed in Sect. 2.2. The employed reference values of the fibre quantities $v_{i}^{\text {ref }}$ to which we wish to optimise are given in Table 2 . The start $\left(\mathrm{CF}_{\mathrm{s}}\right)$ and end values $\left(\mathrm{CF}_{\mathrm{e}}\right)$ of the $\mathrm{CF}$ for these profiles are shown in Table 1. The lowest value of $\mathrm{CF}_{\mathrm{e}}$ obtained by each algorithm is highlighted in bold typeface. The computation time and the number of iterations are specified as well. Here, we distinguish between the computation of the $\mathrm{CF}$ only $(\mathrm{F})$ and of the $\mathrm{CF}$ and its gradient $(\mathrm{F} \& \mathrm{G})$. For profile $(1,0,0)$ no guided mode was found implying that the fundamental mode extends too far into the cladding to be practical.

We observe that all schemes find values for $\mathrm{CF}_{\mathrm{e}}$ that are substantially lower than their starting values $\mathrm{CF}_{\mathrm{s}}$. Further, we note that the stochastic optimisation methods outperform the gradient-based ones when it comes to the lowest value of $\mathrm{CF}_{\mathrm{e}}$. Moreover, these minima are independent of the initial-profile choice. The SA technique attains the same global minimum as the DE scheme, at the expense of about eight times as many function evaluations.

Neither of the Newton algorithms converge to the global minimum. However, they arrive at local minima that are about $6 \%$ to $8 \%$ higher than the global one. An additional optimisation with QN after the MN optimisation does not yield substantially lower values of the CF, and

Table 1 The values $\mathrm{CF}_{\mathrm{S}}$ and $\mathrm{CF}_{\mathrm{e}}$ for various initial profiles for five optimisation parameters, optimised with (combinations of) the MN, QN, SA and DE algorithms

\begin{tabular}{lllllll}
\hline Profile & $\mathrm{CF}_{\mathrm{s}}$ & $\mathrm{CF}_{\mathrm{e}}^{\mathrm{MN}}$ & $\mathrm{CF}_{\mathrm{e}}^{\mathrm{QN}}$ & $\mathrm{CF}_{\mathrm{e}}^{\mathrm{MN} \rightarrow \mathrm{QN}}$ & $\mathrm{CF}_{\mathrm{e}}^{\mathrm{SA}}$ & $\mathrm{CF}_{\mathrm{e}}^{\mathrm{DE}}$ \\
\hline$(1,1,1)$ & 23.6 & $5.44 \mathrm{E}-3$ & $\mathbf{5 . 4 9 E}-\mathbf{3}$ & $5.40 \mathrm{E}-3$ & $\mathbf{5 . 0 9 E}-3$ & $\mathbf{5 . 0 9 E}-\mathbf{3}$ \\
$(1,1,0)$ & 426 & $\mathbf{5 . 3 9 E}-\mathbf{3}$ & $5.54 \mathrm{E}-3$ & $\mathbf{5 . 3 9 E}-\mathbf{3}$ & $5.09 \mathrm{E}-3$ & $5.09 \mathrm{E}-3$ \\
$(1,0,1)$ & 211 & $9.12 \mathrm{E}-2$ & $1.11 \mathrm{E}+1$ & $3.21 \mathrm{E}-2$ & $5.09 \mathrm{E}-3$ & $5.09 \mathrm{E}-3$ \\
$(1,0,0)$ & - & - & - & - & $5.09 \mathrm{E}-3$ & $5.09 \mathrm{E}-3$ \\
$(0,1,1)$ & 23.9 & $9.89 \mathrm{E}-2$ & $5.10 \mathrm{E}-2$ & $9.89 \mathrm{E}-1$ & $5.09 \mathrm{E}-3$ & $5.09 \mathrm{E}-3$ \\
$(0,1,0)$ & $3.1 \mathrm{E} 4$ & $9.83 \mathrm{E}-2$ & $4.98 \mathrm{E}-2$ & $9.83 \mathrm{E}-2$ & $5.09 \mathrm{E}-3$ & $5.09 \mathrm{E}-3$ \\
$(0,0,1)$ & $2.2 \mathrm{E} 3$ & $9.42 \mathrm{E}-2$ & 4.35 & $9.42 \mathrm{E}-2$ & $5.09 \mathrm{E}-3$ & $5.09 \mathrm{E}-3$ \\
\hline F/F\&G & & $0 / 329$ & $403 / 210$ & $50 / 24$ & $2640000 / 0$ & $660000 / 0$ \\
Comp. time & & $5.8 \mathrm{~min}$ & $5.3 \mathrm{~min}$ & $37 \mathrm{~s}$ & 7.0 days & $42.2 \mathrm{~h}$ \\
\hline
\end{tabular}

The computation time and the number of iterations of the $\mathrm{CF}(\mathrm{F})$ and the $\mathrm{CF}$ and its gradient $(\mathrm{F} \& \mathrm{G})$ are shown for the best profile (boldface) 
will therefore no longer be considered. For the SA and DE schemes, there is no improvement upon making an additional QN run, and hence the resulting minima in this parameter space are proper ones. The sensitivity analysis in Sect. 4.4 confirms this. The overall computation times are definitely in favour of the gradient-based routines. The QN algorithm is a little bit faster than the MN scheme, although the latter algorithm attains, in general, somewhat lower minima.

For the best values of $\mathrm{CF}_{\mathrm{e}}$, the fibre quantities $v_{i}$ and their set reference values $v_{i}^{\text {ref }}$ are given in Table 2. Given the choice of the scale factors $\alpha_{i}$, it is not surprising that the values for the dispersion $D$ and mode-field diameter $d_{I I}$, which are about three orders of magnitude larger than the dispersion slope $S$ and the bending loss, correspond to the desired values. The subsequent "competition" between the dispersion slope and the bending loss determines the final minimum. One may adapt the scale factors to reduce the importance of $D$ and MFD $d_{I I}$ such that their end values are within a tolerable region about the desired value so as to arrive at somewhat lower values for the dispersion slope and bending loss. Naturally, this requires a fine tuning of the scale factors, a process for which the Newton algorithms, with their short computation times, are very well suited.

The corresponding optimised profiles and their intensity patterns $S(\rho)$ normalised to the total power carried by the propagating mode are plotted in Fig. 4 at a wavelength of $\lambda=1,550 \mathrm{~nm}$. The Newton algorithms are not able to generate the sharp profile peak near the core/cladding transition. This is due to the fact that during the search the multi-mode regime limitation is encountered. The peak causes the value of the dispersion slope to lower, which can be seen in Fig. 5, where we have performed a wavelength sweep on the four fibre quantities for the best profiles obtained through the MN, QN and DE schemes.

Table 2 Values of the fibre quantities $v_{i}$ with the pertaining scale factors $\alpha_{i}$ at $\lambda=1,550 \mathrm{~nm}$ for which the profile, with five optimisation parameters, has been optimised

\begin{tabular}{lrlllll}
\hline & $v_{i}^{\text {ref }}$ & $\alpha_{i}$ & $v_{i}^{\mathrm{MN}}$ & $v_{i}^{\mathrm{QN}}$ & $v_{i}^{\mathrm{SA}}$ & $v_{i}^{\mathrm{DE}}$ \\
\hline$D(\mathrm{ps} / \mathrm{km} / \mathrm{nm})$ & 13 & 1 & 13.00 & 13.00 & 13.00 & 13.00 \\
$S\left(\mathrm{ps} / \mathrm{km} / \mathrm{nm}^{2}\right)$ & 0 & 1 & $7.20 \mathrm{E}-2$ & $7.27 \mathrm{E}-2$ & $7.01 \mathrm{E}-2$ & $7.01 \mathrm{E}-2$ \\
MFD $d_{I I}(\mu \mathrm{m})$ & 10 & 1 & 10.00 & 10.00 & 10.00 & 10.00 \\
Bend. loss $(\mathrm{dB} / \mathrm{turn})$ & 0 & 1 & $1.43 \mathrm{E}-2$ & $1.41 \mathrm{E}-2$ & $1.35 \mathrm{E}-2$ & $1.34 \mathrm{E}-2$ \\
\hline
\end{tabular}
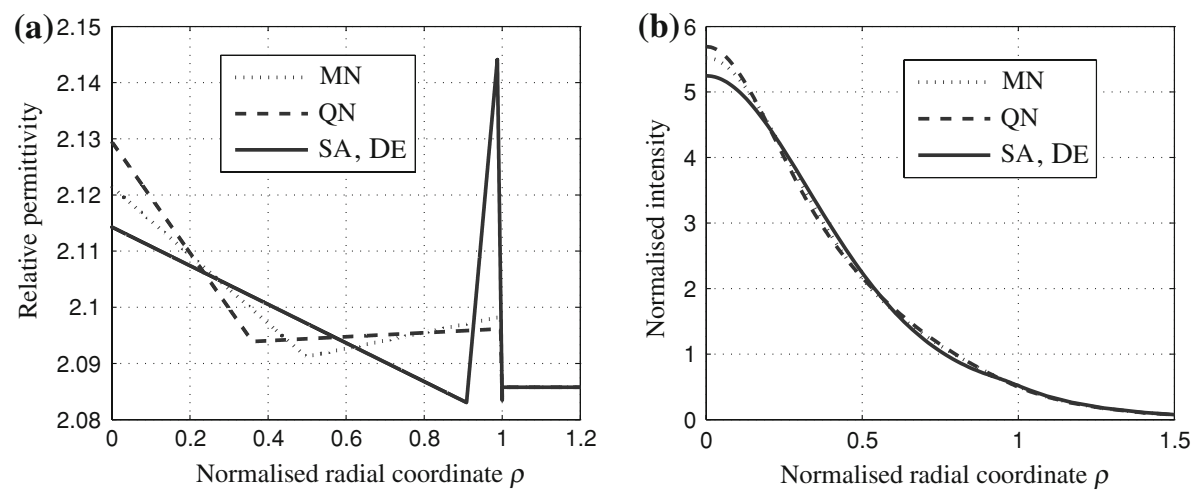

Fig. 4 Best optimised profiles at $\lambda=1,550 \mathrm{~nm}$ (a) and corresponding intensity patterns (b) obtained by optimisation runs with five optimisation parameters 

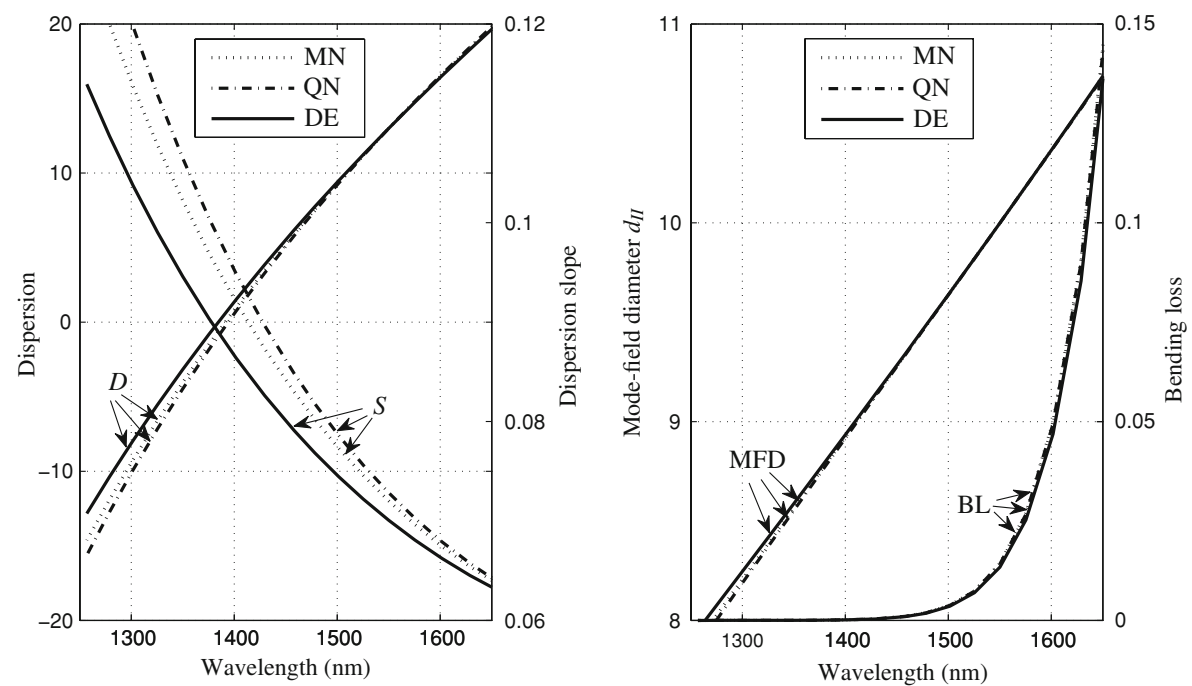

Fig. 5 Dispersion $(D)$, dispersion slope $(S), \operatorname{MFD} d_{I I}$ and bending loss (BL) as a function of wavelength for the best profile obtained by the MN, QN and DE algorithms

\subsubsection{Seven optimisation parameters}

We have also performed optimisation runs with seven optimisation parameters. The results are given in Table 3. Based on the observations in Table 1, we have made a selection in the choice of initial profiles, i.e. profiles starting at $X^{G e}=0 \%$ are excluded due to poor results. We have only shown the results of profiles that contain one transition from $X^{G e}=7 \%$ to $0 \%$.

Again, we observe that optimisation with the Newton algorithms strongly depends on the choice of the initial profile, whereas the DE and SA scheme do not. Mutual differences between the lowest values of the $\mathrm{CF}$ for the various schemes (bold typeface) are marginal (within $1.0 \%$ ). Computation times are much shorter for the Newton algorithms.

The SA scheme performs marginally better than the DE scheme, although at the expense of four times as much computation time. The differences are shown in Table 4, where we have listed the computed fibre quantities that correspond to the best optimised profiles.

Table 3 The values $\mathrm{CF}_{\mathrm{S}}$ and $\mathrm{CF}_{\mathrm{e}}$ for various initial profiles for seven optimisation parameters, optimised with the MN, QN, SA and DE algorithms

\begin{tabular}{llllll}
\hline Profile & $\mathrm{CF}_{\mathrm{s}}$ & $\mathrm{CF}_{\mathrm{e}}^{\mathrm{MN}}$ & $\mathrm{CF}_{\mathrm{e}}^{\mathrm{QN}}$ & $\mathrm{CF}_{\mathrm{e}}^{\mathrm{SA}}$ & $\mathrm{CF}_{\mathrm{e}}^{\mathrm{DE}}$ \\
\hline$(1,1,1,1)$ & 34.5 & $5.78 \mathrm{E}-3$ & $5.66 \mathrm{E}-3$ & $5.09 \mathrm{E}-3$ & $5.09 \mathrm{E}-3$ \\
$(1,1,1,0)$ & 4.1 & $\mathbf{5 . 1 2 E}-\mathbf{3}$ & $\mathbf{5 . 1 6 E}-\mathbf{3}$ & $\mathbf{5 . 0 9 E}-\mathbf{3}$ & $\mathbf{5 . 0 9 E}-\mathbf{3}$ \\
$(1,1,0,0)$ & $8.1 \mathrm{E} 6$ & $5.12 \mathrm{E}-3$ & $5.16 \mathrm{E}-3$ & $5.09 \mathrm{E}-3$ & $5.09 \mathrm{E}-3$ \\
\hline F/F\&G & & $0 / 436$ & $239 / 153$ & $3878000 / 0$ & $952000 / 0$ \\
Comp. time & & $7.7 \mathrm{~min}$ & $5.2 \mathrm{~min}$ & 11.7 days & 2.9 days \\
\hline
\end{tabular}

The computation time and the number of iterations of the $\mathrm{CF}(\mathrm{F})$ and the $\mathrm{CF}$ and its gradient $(\mathrm{F} \& \mathrm{G})$ are shown for the best profile (boldface) 
Table 4 Values of the fibre quantities $v_{i}$ with the pertaining scale factors $\alpha_{i}$ at $\lambda=1,550 \mathrm{~nm}$ for which the profile, with seven optimisation parameters, has been optimised

\begin{tabular}{lrlllll}
\hline & \multicolumn{1}{c}{$v_{i}^{\text {ref }}$} & $\alpha_{i}$ & $v_{i}^{\mathrm{MN}}$ & $v_{i}^{\mathrm{QN}}$ & $v_{i}^{\mathrm{SA}}$ & $v_{i}^{\mathrm{DE}}$ \\
\hline$D(\mathrm{ps} / \mathrm{km} / \mathrm{nm})$ & 13 & 1 & 13.00 & 13.00 & 13.00 & 13.00 \\
$S\left(\mathrm{ps} / \mathrm{km} / \mathrm{nm}^{2}\right)$ & 0 & 1 & $7.02 \mathrm{E}-2$ & $7.06 \mathrm{E}-2$ & $7.01 \mathrm{E}-2$ & $7.01 \mathrm{E}-2$ \\
$\mathrm{MFD} d_{I I}(\mu \mathrm{m})$ & 10 & 1 & 10.00 & 10.00 & 10.00 & 10.00 \\
Bend. loss $(\mathrm{dB} / \mathrm{turn})$ & 0 & 1 & $1.41 \mathrm{E}-2$ & $1.30 \mathrm{E}-2$ & $1.33 \mathrm{E}-2$ & $1.34 \mathrm{E}-2$ \\
\hline
\end{tabular}
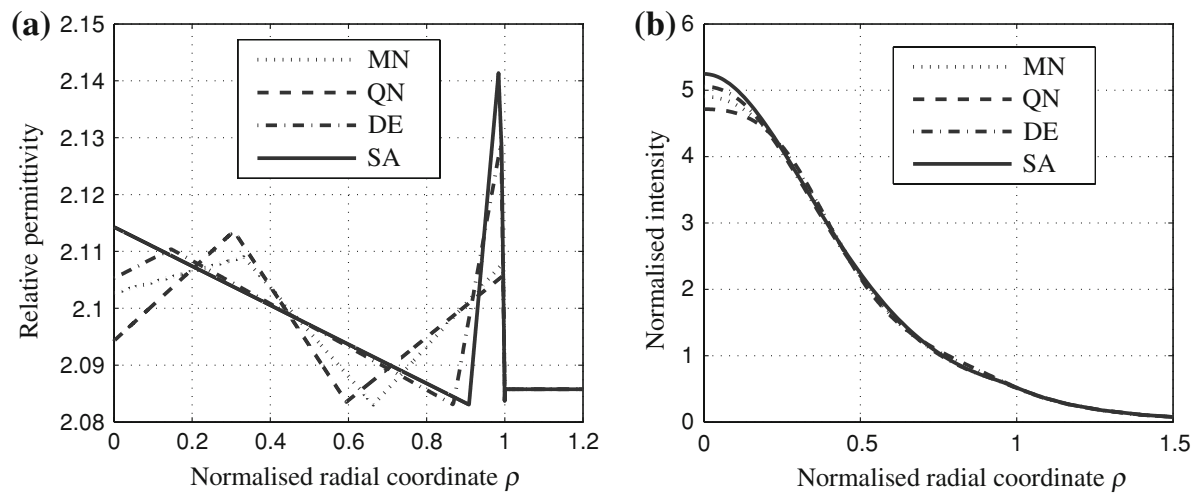

Fig. 6 Best optimised profiles at $\lambda=1,550 \mathrm{~nm}$ (a) and corresponding intensity patterns (b) obtained by the $\mathrm{MN}$ and SA algorithms with seven optimisation parameters

Although the differences in the computed fibre quantities between the various schemes are small, the corresponding optimised profiles, which are plotted in Fig. 6a, are rather different. The corresponding intensity patterns $S(\rho)$ normalised to the total power carried by the propagating mode are plotted in Fig. 6b. In Sect. 4.4, we will present a sensitivity analysis of these optimised profiles, that will give more insight in the influence of each optimisation parameter on the value of the $\mathrm{CF}$.

\subsubsection{More than seven optimisation parameters}

We have carried out similar optimisation runs with 9, 11, 13 and 15 optimisation parameters. To limit the number of initial profiles, we have used the same selection procedure as for the run with seven optimisation parameters. The lowest value of $\mathrm{CF}_{\mathrm{e}}$ for each parameter run with the various schemes are given in Table 5. We point out that in order to achieve such low minima for the DE scheme, we have had to change the control parameter $N P$ to 200. With the initial recommended $N P=8 N$, the attained minima were increasing for an increasing number of optimisation parameters. As a result of these changes, the computation time increases drastically though.

For all algorithms, the value of $\mathrm{CF}_{\mathrm{e}}$ gradually decreases for an increasing number of optimisation parameters, which is good from an optimisation point of view. Still, one has to contemplate on whether this relatively large number of parameters is practical as the 
Table 5 The values $\mathrm{CF}_{\mathrm{e}}$ for the best profiles for nine to fifteen optimisation parameters

\begin{tabular}{llllll}
\hline Algorithm & $\mathrm{CF}_{\mathrm{e}}(9 \mathrm{p})$ & $\mathrm{CF}_{\mathrm{e}}(11 \mathrm{p})$ & $\mathrm{CF}_{\mathrm{e}}(13 \mathrm{p})$ & $\mathrm{CF}_{\mathrm{e}}(15 \mathrm{p})$ & Comp. times \\
\hline MN & $5.09 \mathrm{E}-3$ & $4.96 \mathrm{E}-3$ & $4.96 \mathrm{E}-3$ & $4.91 \mathrm{E}-3$ & $21 / 31 / 54 / 86$ min \\
QN & $5.11 \mathrm{E}-3$ & $5.06 \mathrm{E}-3$ & $5.02 \mathrm{E}-3$ & $5.00 \mathrm{E}-3$ & $7 / 12 / 13 / 15$ min \\
DE & $4.79 \mathrm{E}-3$ & $4.72 \mathrm{E}-3$ & $4.71 \mathrm{E}-3$ & $4.69 \mathrm{E}-3$ & $12 / 62 / 83 / 96$ days \\
SA & $5.09 \mathrm{E}-3$ & $4.98 \mathrm{E}-3$ & $4.97 \mathrm{E}-3$ & $4.81 \mathrm{E}-3$ & $17 / 25 / 31 / 40$ days \\
\hline
\end{tabular}

fabrication process is more involved when the profile consists of more kinks. We remark that profile optimisation with the permittivity as the vertical parameter yields similar results for the simulations performed in this section.

A hybrid method, that first uses a stochastic optimisation scheme to locate the valley that harbours the global minimum followed by a fast Newton one to find it, might prove worthwhile if the computation time of a single $\mathrm{CF}$ iteration is accelerated by several orders of magnitude. For a kick-start using the DE scheme, an acceleration by at least a factor 80 is required to arrive at a total computation time that is within the hour for seven optimisation parameters. We found that a direct numerical integration of our system under the weak guidance approximation (Snyder and Love 1977) only reduces the required acceleration by a factor 1.5 .

\subsection{Profile variations in vertical directions only}

Next, let us consider profile variations in the vertical direction only. This way the size of the parameter space is curtailed drastically. It turns out that the $\mathrm{MN}$ algorithm is most suited because of its short computation times. Further, the results using the MN algorithm turn out to be less dependent on the initial profile choice than those obtained using the QN algorithm. In about $70 \%$ of the possible initial profiles, selected by the procedure that is shown in Fig. 3, the MN algorithm is able to find the global minimum, which has been verified with the DE scheme for up to nine optimisation parameters. In Table 6 , the number of vertical parameters is set against the value of $\mathrm{CF}_{\mathrm{e}}$.

Again, we observe a steady decrease in the value of the $\mathrm{CF}_{\mathrm{e}}$ with a corresponding increase in computation time. Further optimisation with the QN algorithm, while dropping the restriction on the horizontal parameters and thus considering a much larger parameter space, did not yield significantly lower minima. On the whole, the large number of kinks, when optimising for many vertical parameters, renders that option impractical.

Table 6 Values of $\mathrm{CF}_{\mathrm{e}}$ with vertical optimisation parameters only, optimised with the MN algorithm

\begin{tabular}{lllc}
\hline Opt. parameters & $\mathrm{CF}_{\mathrm{e}}$ & Comp. time & \# of iterations \\
\hline 3 & $5.96 \mathrm{E}-3$ & $43 \mathrm{~s}$ & 76 \\
5 & $5.40 \mathrm{E}-3$ & $6 \mathrm{~min}$ & 328 \\
7 & $5.16 \mathrm{E}-3$ & $11 \mathrm{~min}$ & 428 \\
9 & $5.05 \mathrm{E}-3$ & $23 \mathrm{~min}$ & 664 \\
11 & $4.94 \mathrm{E}-3$ & $36 \mathrm{~min}$ & 828 \\
22 & $4.71 \mathrm{E}-3$ & $82 \mathrm{~min}$ & 905 \\
\hline
\end{tabular}


Table 7 Specifications of Corning's SMF-28 $\mathrm{e}^{X B}$ (issued 05/07), and Draka Comteq's ESMF and $\mathrm{BB}^{X S}$ (issued 11/07)

\begin{tabular}{lllllll}
\hline Optical fibre & ESMF & Opt & BB $^{X S}$ & Opt & SMF-28 $\mathrm{e}^{X B}$ & Opt \\
\hline$D(1,550 \mathrm{~nm})$ & $\leq 18$ & 17 & - & 17.3 & $\leq 18$ & 16.8 \\
$D(1,625 \mathrm{~nm})$ & $\leq 22$ & 21.2 & - & 21.7 & $\leq 22$ & 21.1 \\
$\lambda_{D}$ & $1,300-1,322$ & 1,311 & $1,300-1,324$ & 1,313 & $1,302-1,322$ & 1,313 \\
$S\left(\lambda_{D}\right)$ & $\leq 0.090$ & 0.088 & $\leq 0.092$ & 0.090 & $\leq 0.089$ & 0.087 \\
MFD $d_{I I}(1,310 \mathrm{~nm})$ & 9.0 & 8.9 & $8.5-9.3$ & 8.7 & 8.6 & 8.6 \\
MFD $d_{I I}(1,550 \mathrm{~nm})$ & 10.1 & 10.1 & $9.4-10.4$ & 9.9 & 9.8 & 9.8 \\
Bend. loss & & & & & & \\
$-R=25 \mathrm{~mm}, 100$ turns & $\leq 0.05$ & $\mathbf{1 . 1 E - 4}$ & - & - & - & - \\
$-R=10 \mathrm{~mm}, 1$ turn & - & - & $\leq 0.10$ & $\mathbf{0 . 0 6}$ & $\leq 0.50$ & $\mathbf{0 . 1 1}$ \\
\hline
\end{tabular}

Our optimised results are given in the columns denoted by "Opt" next to each fibre

\subsection{Comparison with commercially available fibres}

To see if we can design refractive index profiles such that they meet the specifications of single-mode fibres on today's market, we have extracted the relevant fibre quantities from the data-sheets of two leading optical fibre companies, viz. Draka Comteq: http://www. drakafibre.com/ and Corning: http://www.corning.com/opticalfiber/. In Table 7, these values are listed for several optical fibres, viz. Corning's SMF-28 $\mathrm{e}^{X B}$, Draka Comteq's Enhanced single-mode optical fibre (ESMF), and Draka Comteq's BendBright ${ }^{X S}$ single-mode optical fibre $\left(\mathrm{BB}^{X S}\right)$. We have to set the desired values of the fibre quantities at a wavelength of $\lambda=1,550 \mathrm{~nm}$ in our $\mathrm{CF}$ and we have assumed a core radius of $5 \mu \mathrm{m}$. As the dispersion and dispersion slope are specified in the data-sheets in terms of inequality constraints, we have used the following rational expression (Corning: http://www.corning.com/opticalfiber/)

$$
D(\lambda) \approx \frac{S\left(\lambda_{D}\right)}{4}\left(\lambda-\frac{\lambda_{D}^{4}}{\lambda^{3}}\right) \text { for } 1,200 \mathrm{~nm} \leq \lambda \leq 1,625 \mathrm{~nm},
$$

to arrive at an estimate for $D$ to optimise for. For the zero-dispersion wavelength $\lambda_{D}$, we have used the centre value of the pertaining range, and for the dispersion slope, the maximum allowed value is chosen. Hence, for the ESMF we have found that $D=17 \mathrm{ps} / \mathrm{km} / \mathrm{nm}$, for the $\mathrm{BB}^{X S}, D=17.3 \mathrm{ps} / \mathrm{km} / \mathrm{nm}$, and for the SMF-28e ${ }^{X B}, D=16.8 \mathrm{ps} / \mathrm{km} / \mathrm{nm}$, respectively. For the mode-field diameter $d_{I I}$ of the $\mathrm{BB}^{X S}$, we have chosen the centre value of the given range, viz. MFD $d_{I I}=9.9 \mu \mathrm{m}$. The desired values of the dispersion slope $S$ and the bending loss are set to zero to obtain optimal results. Further, we have made sure that the fibre is still in the single-mode regime for $\lambda_{c e}>1,300 \mathrm{~nm}$ by tweaking the theoretical cut-off frequency in the mode-counting scheme (de Hon and Bingle 2003; de Hon 2003).

After some manual fine-tuning of the scale factors $\alpha_{i}$ in the $\mathrm{CF}$, we have been able to meet the specifications of modern commercially available optical fibres, as is shown in the columns in Table 7 denoted by "Opt". To achieve these results, we have employed nine optimisation parameters and have used both Newton algorithms to optimise with. Although the $\mathrm{BB}^{X S}$ and SMF-28 $\mathrm{e}^{X B}$ have especially been designed to achieve a low macro-bending sensitivity, there still appears to be room for improvement. 
4.4 Sensitivity analysis of the optimised profiles

For the optical-fibre manufacturer, the sensitivity of the fibre quantities, or more specifically of the $\mathrm{CF}$, to small variations in the optimised refractive index profile is of great importance. The less sensitive a profile is, the more robust the manufacturing process will be. To get an idea of the landscape in which the best profiles are located, we have defined distances from the best value of the CF in terms of percentages, i.e $1 \%, 5 \%$ and $10 \%$. Subsequently, we reran the optimisation with the pertaining best optimiser and saved those vectors $x_{n}$ that yield a value of the $\mathrm{CF}$ which is within one of these percentage regions. Since the DE scheme performed best for five optimisation parameters and the SA scheme for seven, we have employed these schemes to determine the regions. This is shown in Fig. 7, where we have shaded, next to the best optimised profile, from dark to light grey the areas that correspond to the $1 \%, 5 \%$ and $10 \%$ deviations from this profile, respectively.

Sensitivity information can also be extracted from the deterministic methods using the gradient vector and the Hessian matrix. Since our MN algorithm provides both on exit, we have employed this scheme to demonstrate the principle. We recall from Sect. 2.1 that the MN algorithm is supplemented with simple bounds and no additional constraints on the optimisation parameters can be added. We have adapted these simple bounds to perform the sensitivity analysis on the profiles obtained by the other schemes.

We employ the simple bounds as defined by Eq. 6, resulting in the optimised MN profiles determined in Sect. 4.1. Due to these bounds, the search for the minimum is generally obstructed and the optimisation may end there. If such a bound $c_{i}$ is actively obstructing the search, the pertaining non-negative Lagrange multiplier $\lambda_{i}$ in the Karush-Kuhn-Tucker (KKT) conditions tells us how sensitive the CF is to a change in such a bound (Nocedal and Wright 1999), i.e. the larger $\lambda_{i}$, the harder the CF is "pushing" or "pulling" against the particular bound. In that case, the following relation holds

$$
\left.\partial_{x_{n}} \mathrm{CF}\left(x_{n}\right)\right|_{x_{n}=x_{n}^{*}}=\sum_{i \in \mathscr{A}\left(x_{n}^{*}\right)} \lambda_{i} \partial_{x_{n}} c_{i}\left(x_{n}^{*}\right),
$$

where the optimisation parameters $x_{n}^{*}$ correspond to the optimal profile, and $\mathscr{A}\left(x_{n}^{*}\right)$ denotes the set of active bounds or constraints. For seven optimisation parameters, Eq. 15 yields
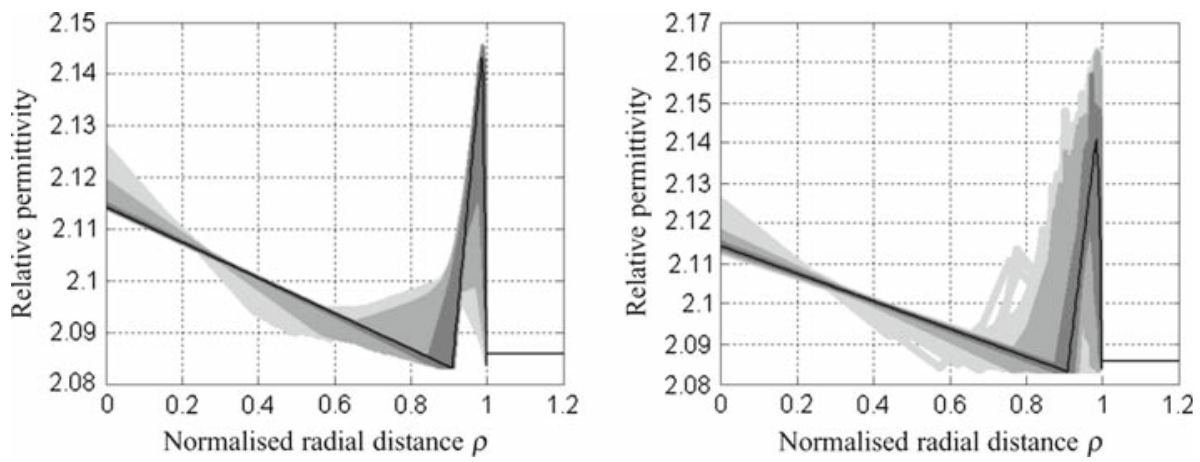

Fig. 7 Best optimised profiles (solid line) obtained by the DE and SA schemes for five (left) and seven (right) parameters, respectively. The dark grey and light grey area denote deviations in the $\mathrm{CF}$ from the best profile by $1 \%, 5 \%$ and $10 \%$, respectively 
Table 8 Deviations $\Delta x_{n}$ from the minimum $x_{n}^{*}$ to achieve an absolute error of $1 \%$ in the value of the CF for the optimised MN profile with seven optimisation parameters

\begin{tabular}{lrrrrrrr}
\hline$n$ & 1 & 2 & 3 & 4 & 5 & 6 & \multicolumn{1}{c}{7} \\
\hline$x_{n}^{*}$ & $3.32 \mathrm{E}-1$ & $3.32 \mathrm{E}-1$ & $3.32 \mathrm{E}-1$ & $6.47 \mathrm{E}-2$ & $8.55 \mathrm{E}-2$ & 0 & $8.21 \mathrm{E}-2$ \\
$\Delta x_{n}$ & $-5.78 \mathrm{E}-3$ & $-5.84 \mathrm{E}-3$ & $-5.72 \mathrm{E}-3$ & $\pm 6.27 \mathrm{E}-5$ & $\pm 3.42 \mathrm{E}-5$ & $6.56 \mathrm{E}-3$ & $\pm 4.31 \mathrm{E}-5$ \\
\hline
\end{tabular}

$$
\left(\begin{array}{c}
-8.9 \\
-8.8 \\
-9.0 \\
0 \\
0 \\
7.8 \\
0
\end{array}\right) \times 10^{-3}=-\lambda_{1}\left(\begin{array}{l}
1 \\
0 \\
0 \\
0 \\
0 \\
0 \\
0
\end{array}\right)-\lambda_{2}\left(\begin{array}{l}
0 \\
1 \\
0 \\
0 \\
0 \\
0 \\
0
\end{array}\right)-\lambda_{3}\left(\begin{array}{l}
0 \\
0 \\
1 \\
0 \\
0 \\
0 \\
0
\end{array}\right)+\lambda_{4}\left(\begin{array}{l}
0 \\
0 \\
0 \\
0 \\
0 \\
1 \\
0
\end{array}\right)
$$

with the following set of active bounds

$$
\begin{array}{ll}
c_{1}: x_{1} \leq(1-\varepsilon) / 3, & c_{2}: x_{2} \leq(1-\varepsilon) / 3, \\
c_{3}: x_{3} \leq(1-\varepsilon) / 3, & c_{4}: x_{6} \geq \varepsilon .
\end{array}
$$

Since all $\lambda_{i}$ are greater than zero, we have found the lowest possible point. The best optimised profile obtained with five optimisation parameters also satisfies the KKT conditions.

Now that we have ascertained that $x_{n}^{*}$ corresponds to the lowest point, we can obtain sensitivity information from the available gradient vector and Hessian matrix $\mathrm{H}$. A Taylor series expansion of the $\mathrm{CF}$ about this point gives us

$$
\mathrm{CF}\left(x_{n}\right)=\mathrm{CF}\left(x_{n}^{*}\right)+\left(\Delta x_{n}\right)^{T} \partial_{x_{n}} \mathrm{CF}+\left(\Delta x_{n}\right)^{T} \mathrm{H}\left(\Delta x_{n}\right)+O\left[\left(\Delta x_{n}\right)^{3}\right],
$$

where $\Delta x_{n}=x_{n}-x_{n}^{*}$. By neglecting third-order derivatives, we obtain for any requested absolute error in the value of CF a quadratic equation in terms of $\Delta x_{n}$. If a bound or constraint is encountered for a certain parameter, a first-order equation suffices as long as the requested absolute error is small. In Table 8, we have shown the deviations in $\Delta x_{n}$ corresponding to an absolute error of $1 \%$ for the optimised $\mathrm{MN}$ profile with seven optimisation parameters.

Apparently, deviations in the optimisation parameter $x_{5}$ prove most stringent, and consequently manufacturing precision is most important there. This parameter is associated with the constriction in the grey sensitivity band in the graph on the right in Fig. 7. As we have assumed first-order equations for those parameters that encounter a bound, the deviations $\Delta x_{1}, \Delta x_{2}, \Delta x_{3}$, and $\Delta x_{6}$ are inversely proportional to the Lagrange multipliers in Eq. 16.

\section{Conclusions}

To aid in the design of single-mode optical fibres, we have implemented and performed automated optimisation of the refractive index profile. The fibre quantities have been used to set a design goal in terms of a cost function. The refractive index profile, or more specifically one of its dopant building blocks, viz. the Germanium concentration profile, has been discretised in continuous piecewise linear segments, whose endpoints serve as the free parameters in the optimisation scheme. We have employed two statistically oriented optimisers, viz. differential evolution and simulated annealing, and two gradient based ones, namely a modified-Newton and a quasi-Newton algorithm. We have discussed their effectiveness in finding the global 
minimum of a cost function. The latter optimisers are fast (minutes), and are therefore the methods of choice for design tools. The statistically oriented optimisers, on the other hand, find the global minimum at the expense of sometimes excessive computation times (days to months). We have found that for a suitable selection of initial profiles the resulting local minima are within $8 \%$ of the global one.

Further, we have performed a sensitivity analysis of the optimised profiles to verify if the attained minima are really the lowest points in the landscape, and to provide the manufacturer with information on how accurate the profile has to be made. A comparison of our optimised fibre designs with commercially available optical fibres demonstrates that there is still room for improvement in the fibre market.

Open Access This article is distributed under the terms of the Creative Commons Attribution Noncommercial License which permits any noncommercial use, distribution, and reproduction in any medium, provided the original author(s) and source are credited.

\section{References}

Bingle, M., de Hon, B.P., van Stralen, M.J.N.: Electromagnetic modelling and optimisation for the design of single-mode optical fibres. In: 2001 URSI International Symposium of Electromagnetic Theory, vol. 307.2/MN06.20, pp. 515-517. Victoria, CA, 13-17 May 2001

de Hon, B.P.: A modal impedance-angle formalism: rigorous proofs for optical fiber mode counting and bracketing. Radio Sci. 38(2), 8013 (2003)

de Hon, B.P., Bingle, M.: A modal impedance-angle formalism: schemes for accurate graded-index bent-slab calculations and optical fiber mode counting. Radio Sci. 38(2), 8012 (2003)

Dil, J.G., Blok, H.: Propagation of electromagnetic surface waves in a radially inhomogeneous optical waveguide. Opt. Quant. Electron. 5, 415-428 (1973)

Fletcher, R.: A new low rank quasi-Newton update scheme for nonlinear programming. In: Ceragioli, F., Dontchev, A., Furuta, H., Marti, K., Pandolfi, L. (ed.) IFIP International Federation for Information Processing, System Modeling and Optimization, vol. 199, pp. 275-293. Springer, Boston (2006)

Fletcher, R., Leyffer, S.: User manual for FilterSQP. University of Dundee Numerical Analysis Report NA/181. http://www-neos.mcs.anl.gov/. April 1998, version1, June 1998, updated March 1999

Gill, P.E., Murray, W.: Numerical Methods for Constrained Optimisation. Academic Press, London (1974)

Goffe, W., Ferrier, G., Rogers, J.: Global optimization of statistical functions with simulated annealing. J. Econom. 60(1/2), 65-100 (1994). http://www.netlib.org/opt/simann.f/

Hermann, W., Wiechert, D.U.: Refractive index of doped and undoped PCVD bulk silica. Mat. Res. Bull. 24(9), 1083-1097 (1989)

Kirkpatrick, S., Gelatt, C.D., Vecchi, M.P.: Optimization by simulated annealing. Science 220(4598), 671680 (1983)

Meyberg, K., Vachenauer, P.: Höhere Mathematik. Springer, Berlin (1991)

Mishra, S.K.: Global optimization by differential evolution and particle swarm methods: evaluation on some benchmark functions (2006a). http://www.ssrn.com/abstract=933827. Accessed 30 September 2006

Mishra, S.K.: Performance of the barter, the differential evolution and the simulated annealing methods of global optimization on some new and some old test functions (2006b). http://www.ssrn.com/ abstract $=941630$. Accessed 1 November 2006

Nocedal, J., Wright, S.J.: Numerical Optimization. Springer-Verlag, New York (1999)

Numerical Algorithms Group Ltd.: NAG Fortran Library Mark 20 (2001). http://www.nag.co.uk/

Rahmat-Samii, Y., Michielssen, E. (eds.): Electromagnetic Optimization by Genetic Algorithms. Wiley Series in Microwave and Optical Engineering. Wiley, New York (1999)

Smink, R.W., de Hon, B.P., Tijhuis, A.G.: Bending loss in optical fibers-a full-wave approach. J. Opt. Soc. Am. B 24(10), 2610-2618 (2007)

Snyder, A.W., Love, J.D.: Optical Waveguide Theory. Chapman and Hall, London (1977)

Storn, R., Price, K.: Differential evolution—a simple and efficient heuristic for global optimization over continuous spaces. J. Global. Optim. 11(4), 341-359 (1997)

Weile, D., Michielssen, E.: Genetic algorithm optimization applied to electromagnetics: a review. IEEE Trans. Antennas Propag. 45(3), 343-353 (1997) 